\section{JTI}

JOURNAL OF

TRAUMA AND INJURY

\title{
Rare Imaging of Fat Embolism Seen on Computed Tomography in the Com- mon Iliac Vein after Polytrauma
}

\author{
Hojun Lee, M.D., Jonghwan Moon, M.D., Junsik Kwon, M.D., \\ John Cook-Jong Lee, M.D. \\ Division of Trauma Surgery, Department of Surgery, Ajou University School of Medicine, \\ Suwon, Korea
}

Received: June 2, 2018

Revised: July 31, 2018

Accepted: August 13, 2018

\section{Correspondence to}

John Cook-Jong Lee, M.D.

Division of Trauma Surgery, Department of Surgery, Ajou University School of Medicine, 164 Worldcup-ro, Yeongtong-gu,

Suwon 16499 , Korea

Tel: $+82-31-219-7492$

Fax: +82-31-219-7781

E-mail: ajoutraumacenter@gmail.com

Fat embolism refers to the presence of fat droplets within the peripheral and lung microcirculation with or without clinical sequelae. However, early diagnosis of fat embolism is very difficult because the embolism usually does not show at the computed tomography as a large fat complex within vessels. Forty-eight-year-old male with pedestrian traffic accident ransferred from a local hospital by helicopter to the regional trauma center by two flight surgeons on board. At the rendezvous point, he had suffered with dyspnea without any airway obstruction sign with $90 \%$ of oxygen saturation from pulse oximetry with giving $15 \mathrm{~L}$ of oxygen by a reserve bag mask. The patient was intubated at the rendezvous point. The secondary survey of the patient revealed multiple pelvic bone fracture with sacrum fracture, right femur shaft fracture and right tibia head fracture. Abdominal computed tomography was performed in 191 minutes after the injury and fat embolism with Hounsfield unit of -86 in his right common iliac vein was identified. Here is a very rare case that mass of fat embolism was shown within common iliac vein detected in computed tomography. Early detection of the fat embolus and early stabilization of the fractures are essential to the prevention of sequelae such as cerebral fat embolism.

Keywords: Multiple trauma; Embolism, Fat; Femoral fractures; Hip fractures

This is an Open Access article distributed under the terms of the Creative Commons Attribution Non-Commercial License (http://creativecommons.org/licenses/by-nc/4.0/) which permits unrestricted noncommercial use, distribution, and reproduction in any medium, provided the original work is properly cited. 


\section{JTI}

\section{INTRODUCTION}

Fat embolism refers to the presence of fat droplets within the peripheral and lung microcirculation with or without clinical sequelae [1]. However, early diagnosis of fat embolism is very difficult because the embolism usually does not show at the computed tomography (CT) as a large fat complex within vessels. We share a rare case of fat embolism seen on CT in the common iliac vein after the injury.

\section{CASE REPORT}

The patient is 48-year-old male with pedestrian traffic accident transferred from a local hospital by helicopter with our medical staff including two flight surgeons on board. At the rendezvous point, the patient was intubated by one of our medical staff because of his medical condition presented with $90 \%$ of oxygen saturation on pulse oximetry with deterred mental status. This patient arrived

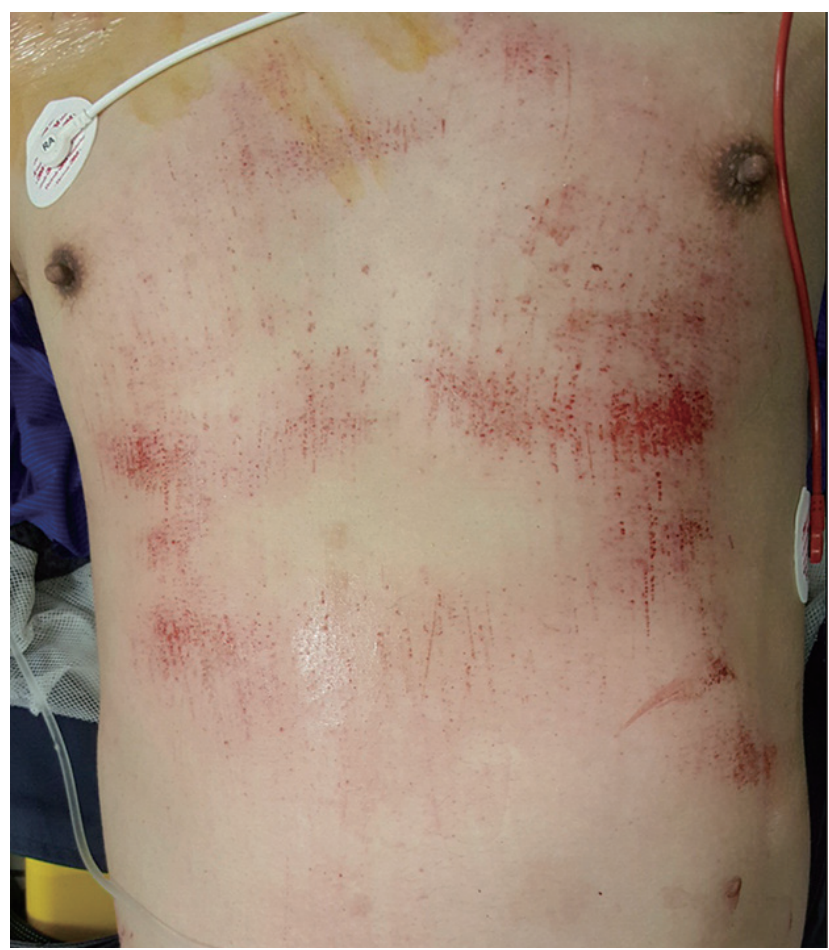

Fig. 1. Presence of abrasion on upper trunk makes more difficult to distinguish from the petechial rash which is one of signs of fat embolism syndrome (FES).

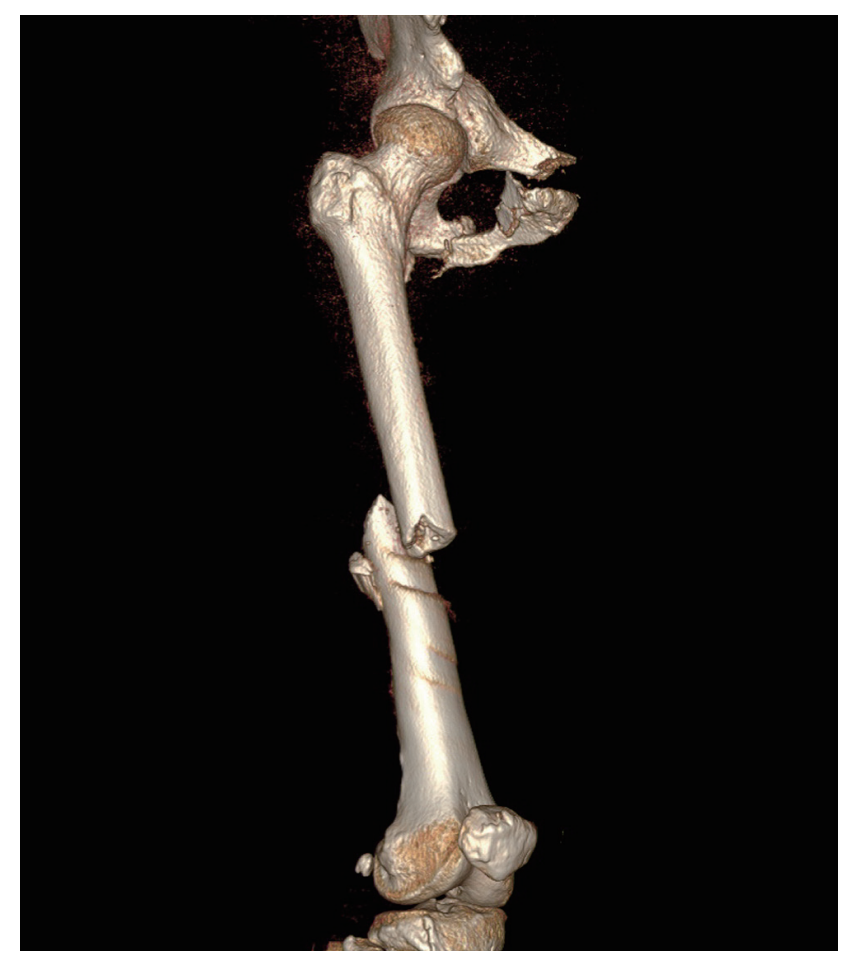

Fig. 2. Femur computed tomography reveals fracture on the shaft of his right femur.

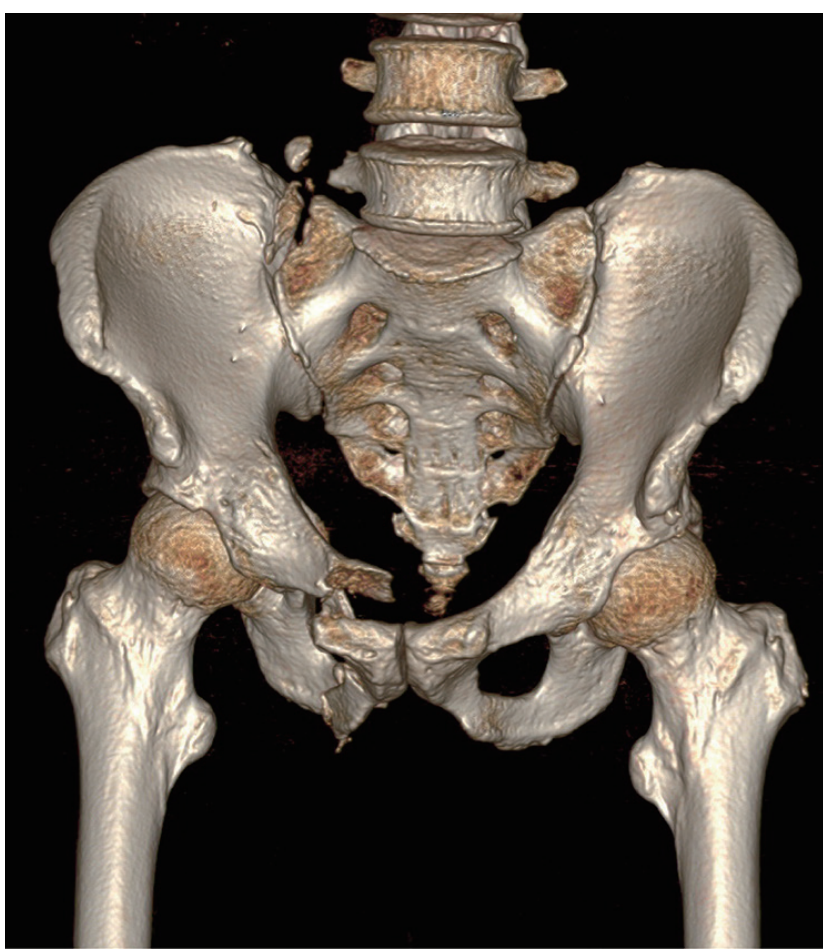

Fig. 3. Pelvis computed tomography shows multiple pelvic bone fracture with sacrum fracture. 
to our trauma center 114 minutes after the estimated time of the injury. Secondary survey revealed $8 \mathrm{~cm}$ in

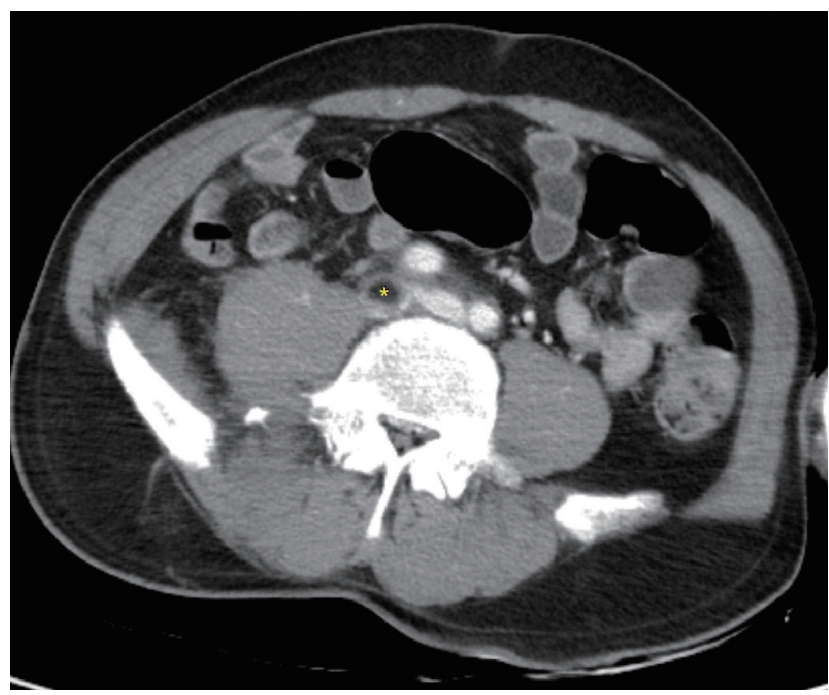

Fig. 4. Fat embolism in his right common iliac vein was shown on the axial plane of abdominal computed tomography. In order to determine whether the embolism is consistent with fat, Hounsfield unit measured at where Asterisk symbol on this figure showed -86 Hounsfield units.

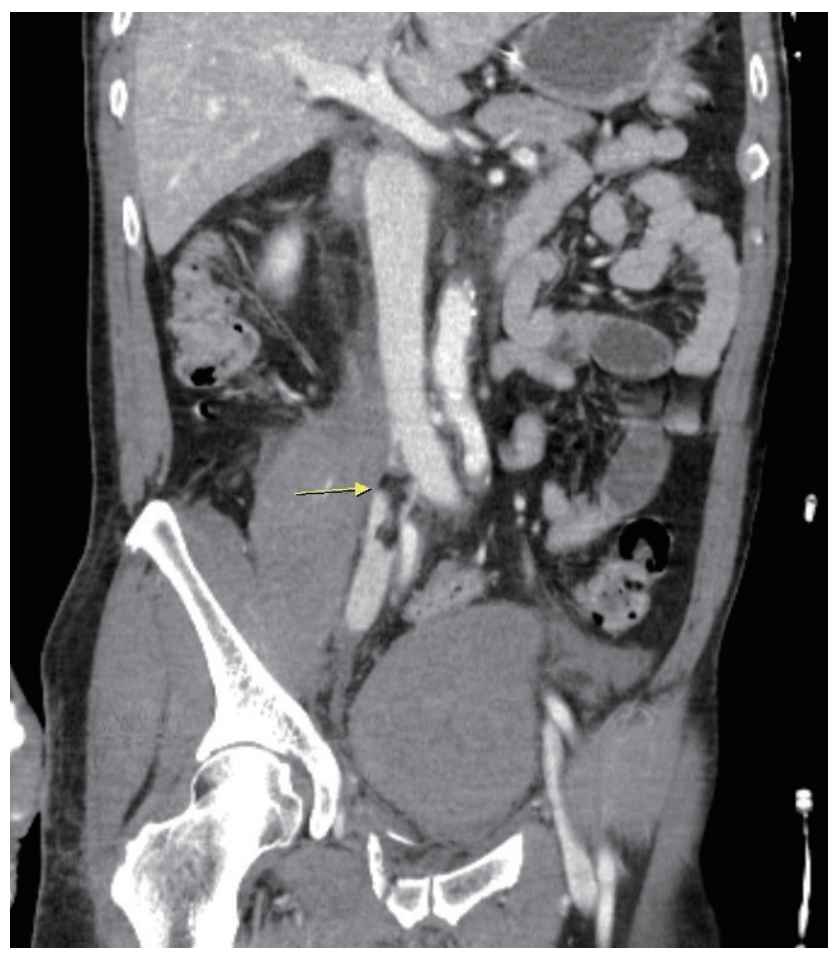

Fig. 5. Fat embolism in his right common iliac vein was shown on the coronal plane of abdominal computed tomography (arrow indicated). length of scalp laceration, extensive abrasion on anterior upper trunk (Fig. 1) and right femur shaft fracture (Fig. 2). Initial radiologic findings taken from the trauma bay revealed multiple facial bone fractures including maxilla, nasal bone and orbit wall, skull fracture with Subarachnoid hemorrhage, multiple long bone fractures in his low extremity such as right femur shaft fracture and right tibia head fracture, and multiple pelvic bone fracture with sacrum fracture (Fig. 3). Due to the pelvic bone fracture, we performed enhanced CT on his abdomen and pelvis. An embolism with -86 Hounsfield Unit consistent with fat in his right common iliac vein was seen in the CT (Figs. 4, 5). CT Pulmonary angiography was checked after 6 hours from initial computed tomography with contrast enhancement and there was no evidence of pulmonary embolism. At hospital day 1, inferior vena cava (IVC) filter was inserted (Fig. 6). At hospital day 2, fractures in his right tibia and right femur was stabilized by orthopedic operations. The IVC filter was successfully removed at hospital day 18 after confirmation of the complete resolution of the embolism by contrast enhanced CT. He left hospital without any event of fat embolism syndrome (FES).

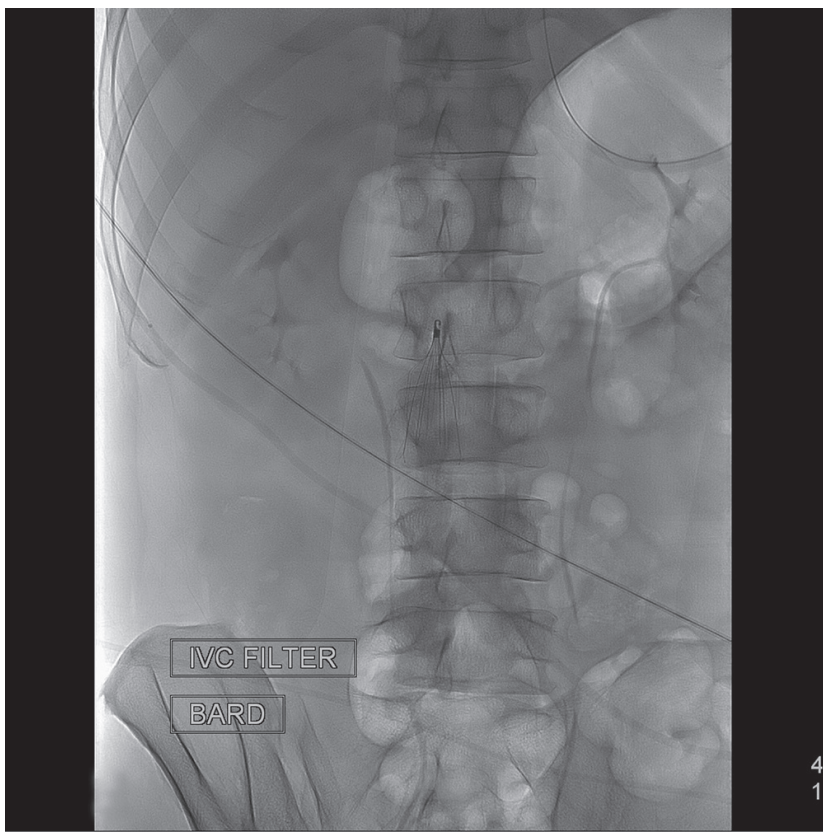

Fig. 6. IVC filter was placed in order to prevent fat embolus migration. IVC: inferior vena cava. 


\section{DISCUSSION}

FES is a multisystem disease most often involving lungs, brain, and skin such as petechiae [2]. Fat embolism is often challenging to make diagnosis within 24 hours of injury because it manifests key symptoms after 24 to 72 hours of injury [3]. Since the fat globules are too large to penetrate capillary, initial symptoms of FES are believed to be caused by mechanical occlusion of blood vessels with fat globules [4]. In this rare case, fortunately, the patient was diagnosed with fat embolism by less than 4 hours after the injury. In addition, this patient has extensive abrasion on his anterior thorax that makes trauma surgeons difficult to find petechial rash development as key sign of the FES. This patient was triaged appropriately to the trauma center for the thorough evaluation from top to bottom fashion including focused assessment with sonography in trauma and abdominal CT with enhancement. Even positive finding on the focused assessment with sonography in trauma facilitated us to make easier decision to check his abdomen with contrast enhanced $\mathrm{CT}$. Although retrospective review of the pelvic CT without contrast enhancement showed suspicious finding of fat embolism, it would be missed without the enhanced CT. In most cases of FES, FES resolves spontaneously. Therefore, this may be a controversial issue about placing the IVC filter when the fat embolus was seen at inferior vena cava or other veins in low extremities because there is no such treatment guideline for this rare condition of "gross fat embolism" to follow. Some physicians may not hesitate to place the retrievable IVC filter in similar cases for prevention of fat embolization [5]. However, I found another rare case report of fat embolus within distal superficial femoral vein seen in CT angiogram after trauma. In this another rare case, size of the embolus seems to be smaller than our case though, the patient was recovered completely without placing the IVC filter [6]. Because of limited number of cases, there is no answer yet for whether placing the IVC filter for fat embolus seen on CT. However, for trauma patients, especially with low extremity long bone fractures, trauma surgeons and radiologists must closely review low extremity veins and IVC thoroughly and realize fat embolism may be seen in the CT angiogram because early detection of the embolus and early stabilization of the fractures are essential to the prevention of sequelae such as cerebral fat embolism.

\section{REFERENCES}

1. Akhtar S. Fat embolism. Anesthesiol Clin 2009;27:533-50.

2. Johnson MJ, Lucas GL. Fat embolism syndrome. Orthopedics 1996;19:41-8; discussion 48-9.

3. Shaikh N. Emergency management of fat embolism syndrome. J Emerg Trauma Shock 2009;2:29-33.

4. Riding G, Daly K, Hutchinson S, Rao S, Lovell M, McCollum C. Paradoxical cerebral embolisation. An explanation for fat embolism syndrome. J Bone Joint Surg Br 2004;86:95-8.

5. Cornman-Homonoff J, Li D, Schiffman M. Pre-operative renal artery embolization and suprarenal IVC filter placement for prevention of fat embolization in renal angiomyolipoma with venous extension. Clin Imaging 2017;43:24-7.

6. Chowdhary V, Mehta V, Bajaj T, Scheiner J. Rare imaging of a known entity: fat embolism seen on CT in lower extremity vein after trauma. Radiol Case Rep 2017;12:488-90. 